\title{
CORRIGENDA
}

\section{CRTAP mutations in lethal and severe osteogenesis imperfecta: the importance of combining biochemical and molecular genetic analysis}

Fleur S Van Dijk, Isabel M Nesbitt, Peter GJ Nikkels, Ann Dalton, Ernie MHF Bongers, Jiddeke $M$ van de Kamp, Yvonne Hilhorst-Hofstee, Nicolette S Den Hollander, Augusta MA Lachmeijer, Carlo L Marcelis, Gita MB Tan-Sindhunata, Rick R van Rijn, Hanne Meijers-Heijboer, Jan M Cobben and Gerard Pals

European Journal of Human Genetics (2009) 17, 1692; doi:10.1038/ejhg.2009.137

Correction to: European Journal of Human Genetics (2009) 17, 1560-1569; doi:10.1038/ejhg.2009.75; published online 24 June 2009

Since the publication of the above paper, the authors have identified two errors:

- In the Discussion (p 10), Table 2 (p 9) and Supplementary Figure 6, the mutation c.404delC should be c.404delG.
- In the pedigree depicted of family 1 there also is an error since VI: 5 is still alive.

The authors would like to apologise for these mistakes.

\section{Caveolinopathies: from the biology of caveolin-3 to human diseases}

Elisabetta Gazzerro, Federica Sotgia, Claudio Bruno, Michael P Lisanti and Carlo Minetti

European Journal of Human Genetics (2009) 17, 1692; doi:10.1038/ejhg.2009.147

Correction to: European Journal of Human Genetics advance online publication, 8 July 2009; doi:10.1038/ejhg.2009.103

Since the publication of their paper the authors have noticed that the reference cited as 7 on page 2 of their paper is incorrect. The correct reference for this citation is:

Murphy RM, Mollica JP, Lamb GD. Plasma membrane removal in rat skeletal muscle fibers reveals caveolin-3 hot-spots at the necks of transverse tubules. Exp Cell Res 2009; 315: 1015-1028.

In addition, the other citation of reference 7 on page 8 should instead refer to reference 6 .

The authors would like to apologize for any inconvenience caused. 